\title{
Prevalência de deficiência de ferro em gestantes de primeira consulta em centros de saúde de área metropolitana, Brasil. Etiologia da anemia
}

\author{
The prevalence of iron deficiency in pregnant women at their first consultation in \\ health centers in a metropolitan area, Brazil. Etiology of anemia in anemic \\ pregnant women
}

\author{
Elvira Maria Guerra*, Orlando Cesar de Oliveira Barretto**, Aymoré Vaz Pinto** , Kátia Gomes \\ Castellão*
}

\begin{abstract}
GUERRA, E. M. et al. Prevalência de deficiência de ferro em gestantes de primeira consulta em centros de saúde de área metropolitana, Brasil. Etiologia da anemia. Rev. Saúde públ., S. Paulo 26: $88-95,1992$. No período compreendido entre abril e outubro de 1988 , foram estudadas 363 gestantes de primeira consulta, que estavam inscritas no Programa de Atendimento à Gestante em oito Centros de Saúde da Secretaria da Saúde do Estado de São Paulo (Brasil). Na ocasião da coleta de material estas gestantes não faziam uso de medicamentos que continham ferro, ácido fólico, vitamina $B_{12}$ ou associações destes. A idade média das gestantes foi de 25 anos; $65,9 \%$ delas pertenciam a famílias com renda de até um SMPC (salário mínimo per capita) e apenas 3,1\% pertenciam a famílias com renda superior a 3 SMPC. Tomando-se a saluração da transferrina inferior a $15 \%$ como índice mínimo para definir a deficiência de ferro, a prevalencia de deficiência de ferro no primciro trimestre $(4,6 \%)$ foi significativamente menor do que a observada no segundo $(17,3 \%)$, e esta foi menor do que no tereciro trimestre $(42,8 \%)$. A prevalencia de deficiência de ferro lotal agrupada nos três trimestres foi de $12,4 \%$. Não houve diferença significativa entre as prevalencias de deficiência de ferro segundo o númcro de partos. Esta prevalencia foi maior no grupo das gestantes que pertenciam a famílias com renda de até $0,5 \mathrm{SMPC}$. Nas gestanıes anêmicas, $46,7 \%$ eram deficientes de ferro, $44,4 \%$ de ácido fólico, $20,0 \%$ de ferro e ácido fólico e nenhuma delas eram deficientes de vitamina $B_{12}$.
\end{abstract}

Descritores: Deficiência de ferro, epidemiologia. Complicações na gravidez. Anemia hipocrômica, etiologia.

\section{Introdução}

A gestação representa para mulher um estado de sobrecarga físiológica, pois o desenvolvimento do feto não só se sobrepóc ao melabolismo materno, como também o modifica susbstancialmente. Detcrmina cntão, um reajuste do organismo materno a csla nova condição ${ }^{1,51}$.

$\mathrm{Na}$ gestaçăo ocorre normalmente uma hemodiluição decorrente do maior aumento do volume plasmático (aproximadamente $50 \%) \mathrm{cm}$ relaç̌̃o do volume critrocitário (de 20-30\%), com o pico des-

\footnotetext{
* Instituto Adolfo Lutz. Divisāo de Patologia - Seção de Fematologia - São P'aulo, SP' - Brasil.

* Faculdade de Medicina da Universidade de São Paulo Laboratório de Pesquisas Hematológicas - São Paulo, $S P-13$ rasil.

*** Faculdade de Medicina da Universidade de Brasúlia Brasília, DF - Brasil
}

Separatas/Reprints: E.M. Guerra - Av. Dr. Amaldo, 355 01246-902 - São Paulo, SP, Brasil

Publicação financiada pela I‘APLSP. Proecesso Saúde Coletiva 91/4994-0 ta hemodiluição por volta do sćtimo mês de gestação, 25, 32, 33, 36, 38 .

Quando a gravide\% ocorre em mulheres com reservas suficientes para atender às necessidades da formação da placenta e do felo, o organismo materno mantém-se em equilíbrio, não acontccendo o mesmo com aquelas que apresentam poucas reservas de ferro, ácido fólico, vitamina $\mathrm{B}_{12}$, e que facilmente se tornarão anêmicas durante a gravidez.

A clevada incidência de anemia nutricional em gestantes pode estar relacionada com a dieta deficiente c/ou pelo aumento da demanda de ferro, ácido fólico e vitamina $B_{12}{ }^{15,26,27,40,45}$. Na gravidez há um aumento das necessidades de ferro em torno de $1.000 \mathrm{mg}$ para suprir a expansão da massa critrocilíria da própria gestante, a lormação do sangue da placenta c do feto c ainda para compensar as perdas durante o parto 5 .

A dicta normal nem sempre é suficiente para suprir as necessidades de ferro na gestação, sendo necessário alé dois anos para restabelecer os depósitos perdidos no ciclo gravídico-puerperal. Tem sido por isso proposta suplementação de ferro durante a ges- 
tação, mesmo em mulheres normais $1,8,35,40,46$.

Segundo Herbert ${ }^{18}$ a deficiência de ferro ocorre em três fases: na primeira ocorre um decréscimo dos estoques de ferro que é rellclido pela queda da ferritina sérica $(<12 \mu \mathrm{g} / \mathrm{L})$. Na segunda, a protoporfirina está elevada, a saturação da transforrina está reduzida e em 95\% dos casos a hemoglobina está normal. Só então na terceira fase ocorre a anemia com a queda da concentração de hemoglobina, apresentando hemácias microciticas e hipocrômicas. Estas três fases propostas por Herbert não são estálicas, podendo haver a presença de microcilose e hipocromia na segunda fase antecedendo a anemia.

No Brasil a anemia por deficiência de ferro tem sido estudada por diversos autores ${ }^{29}, 37,41,42,45$, e os resultados encontrados tem variado segundo: os critérios de seleção da amostragem; os critérios adotados para caracterizar a deficiência de ferro; a idade gestacional; as condições sócio-econômicas das gestantes; o estado de sancamento básico da região estudada.

A proposição deste trabalho foi de estudar a prevalência de deficiência de ferro $\mathrm{cm}$ gestantes de primeira consulta na rede estadual no subdistrito do Butantan, bem como estudar a frequiencia de deficiência de ferro, ácido fólico e vitamina $B_{12}$ nas gestantes anêmicas. Para caracterizar lais deficiências, foram adotados os critćrios da Organização Mundial da Saúde.

\section{Material e Método}

\section{Amostragem}

Do total de 481 gestantes de primeira consulta, inscritas no Programa de Alendimento à Gestante, em oito Centros de Saúde da Secretaria da Saúde do Estado, no Subdistrito de Paz do Butantan, situado na zona oeste do Município de São Paulo, apresentando grande númcro de lavelas ${ }^{11} \mathrm{c}$ inlra-estrutura básica carente ${ }^{39}$. No período comprecndido entre abril a outubro de 1988 foram estudadas 363 gestantes, pois o restante fazia uso de medicamentos que continham ferro, ácido fólico, vitamina $B_{12}$ ou associações desıcs, o que as excluiu do cstudo.

A Secretaria da Saúde do Estado de São Paulo mantém o Programa de Alendimento à Gestante nos Centros de Saúde, no qual são solicitados, na inscrição ou na primeira consulta médica, exames laboratoriais de rotina ${ }^{40}$. Todas as gestantes que procuraram o serviço pela primcira $\mathrm{vez}$, naçucla gestação, nos Centros de Saúde*, foram agendadas

* Escola Butantan, II do Butantan, III São Luiz, II do Rio Pequeno, II do Caxingui, $\amalg$ Vila Borges, III Monte Kemel \& II Real l'arque. para colcta de material para exames laboratoriais, bem como para aplicação de inquérito.

Foi aplicado um questionário à população estudada, contendo as seguintes informaçōes: idade da gestante, idade gestacional, paridade, intervalo do último parto, uso de medicamentos, renda familiar e número de pessoas que compunham a familia da gestantel ${ }^{16}$.

Foram colhidas amostras de sangue para as dosagens de: hemoglobina, ferro sérico, capacidade total de ligação com o ferro (CTLF), saturação da transferrina, ácido fólico e vitamina $B_{12}$. $\mathrm{Na}$ mesma ocasião foram recebidas amostras de fezes das gestantes.

\section{Metodologias Laboratoriais}

Foram utilizados os seguintes métodos: - dosagem de hemoglobina pelo método da cianomelahemoglobina?;

- dosagem de ferro sérico e capacidade total de ligação com o ferro (CTLF) pelo mélodo da batofenantrolina $a^{3}, 22,30$, com os valores do ferro sérico e capacidade total de ligação com ferro, calculouse a percentagem da saluração da transferrina; - dosagem da vitamina $B_{12}$ pelo método microbiológico ${ }^{31,44}$ utilizando o Lactobaccillus leischmannii ATCC 7830, somente para as gestantes anêmicas; - dosagem de ácido fólico através do método microbiológico ${ }^{47}$, utilizando o Lactobaccillus casei ATCC 7469, somente nas gestantes anêmicas; - exame protoparasitológico através dos métodos: dircto, Holfman ${ }^{20}$, Kato $^{23}$ e Willis ${ }^{48}$.

\section{Critérios de Avaliação}

1. Considerou-se a gestunte anêmica quando a concentração de hemoglobina foi inferior a $11,6 \mathrm{~g} /$ $\mathrm{dl}$, pois estando o Município de São Paulo a $835 \mathrm{~m}$ do nível do mar, acrescenlou-se $0,2 \mathrm{~g} / \mathrm{dl}$ à concentração de $11 \mathrm{~g} / \mathrm{dl}^{49}$, para cada $300 \mathrm{~m}$ de altitude ${ }^{12,21}$.

2. A Organização Mundial da Saúde ${ }^{15,50}$ considera haver deficiência de ferro $\mathrm{cm}$ gestantes quando a concentração de ferro sérico for inferior a $50 \mu \mathrm{g} / \mathrm{dl}$ ou saluração da transferrina inferior a 15\%; deficiência de ácido fólico quando a concentração de ácido fólico sérico lor inferior a $3 \mathrm{ng} / \mathrm{ml}$; deficiência de vilamina $B_{12}$ quando a concentração sérica desta vitamina for inferior a $80 \mathrm{pg} / \mathrm{ml}$. No presente trabatho foram adolados os critérios da Organização Mundial de Saúde para caracterizar deficiência de ferro quanto alos dois critérios anteriormente citados.

3. Na variável paridade não foram considerados os abortos tidos pela gestante, mas foram considerados os casos de natimortos.

4. Na variável renda foi utilizado o SMPC (salário mínimo per capita), o qual foi obtido atra- 
vés de duas divisões. Primciro, foi dividida a renda familiar pelo piso nacional de salários do mês da aplicação do questionário. A seguir, a renda familiar em salários mínimos foi dividida pelo número de pessoas que compunham a família da gestante, inclusive as crianças, obtendo-sc assim o SMPC. O valor do piso nacional de salário foi dividido pelo valor oficial do dólar americano no dia 30 de cada mês, obtendo-se assim, o valor de um piso nacional de salário nessa moeda.

\section{Análise dos Dados}

As frequiências foram obtidas atravćs do programa de banco de dados DBASE III PLUS para microcomputador IBM PC XT compalivel. A análise estatística foi rcalizada através dos seguintes testes:

- teste do Qui-quadrado para comparar as prevalências de deficiência de ferro $\mathrm{cm}$ relação ao trimestre gestacional, paridade, intervalo do úlumo parto e renda familiar;

- análise de variância para comparar as médias das dosagens de ferro sérico, capacidade lowal de ligação com ferro, saturação da transferrina nos três trimestres gestacionais. Quando a análise mostrou diferença estalística usou-se o Teste de Scheffe como teste complementar da análise de variância.

O nível de significância aceito foi igual ou menor do que $0,05(\mathrm{p}<0,05)$.

\section{Resultados e Discussão}

A idade média das gestantes foi de 25 anos, com idade mínima de 14 c máxima de 46 anos. Não houve diferença significativa entre as médias das idades das gestantes nos três trimestres de gestação ${ }^{17}$ mostrando ser a amostra homogênea quanto a este aspecto.

Das 363 gestantes, $188(51,8 \%)$ cram caucasóides e $175(48,2)$ negróides. Não encontramos nenhuma gestante mongolóide no grupo estudado, provavelmente devido às características sócioeconômicas desse grupo. Apenas 33,6\% das gestantes eram nascidas no Estado de São Paulo; $11,6 \%$ eram procedentes de outros Estudos da regiño sudeste, $44,6 \%$ da região nordeste, $8,8 \%$ da região sul, $1,1 \%$ da região centro-ocste do Brasil e apenas uma $0,3 \%$ cra proveniente do Chile, caractcrizando uma população de migrantes de outros Estados.

Das gestanles que declararam renda familiar $(n=358), 65,9 \%$ pertenciam a famílias com renda até 1,0 SMPC, 31,0\% a famílias com renda maior que 1,0 até 3,0 SMPC c apenas 3,1\% pertenciam a familias com renda superior a 3,0 SMPC. O salário mínimo per capita médio foi de 0,98 . No período de abril a outubro de 1988 , um piso nacional de salário correspondia, cm média, a US\$ 52,3 , tendo variado de US\$ 50,1 a US\$53,5.

A maior parte das gestantes (54\%) procurou o Scrviço de Pré-Natal no primeiro trimestre de gestação, 38,3\% no segundo e apenas $7,7 \%$ no terceiro trimestre. Entre o total de gestantes de terceiro trimestre, de primcira consulta, que procuraram os Centros de Saúde, a maior parte fazia uso de medicamentos contendo ferro, ácido fólico, vitamina $\mathrm{B}_{12}$, o que explica o número menor de gestantes em rclação acs outros trimestres, além do fato delas procurarem os serviços de saúde logo no início da gestação, possivelmente devido a grande oferta de serviços na região do subdistrito do Butantan ${ }^{17}$.

As médias da concenuraçāo de ferro sérico, capacidade total de ligação com ferro e saturação da transforrina são apresentadas na Tabela 1.

Os valores do ferro sérico e da saturação da transferrina tiveram decréscimo significativo com o decorrer da idade gestacional, enquanto que a capacidade total de ligação com ferro teve aumento significativo, conlïrmando que no tercciro trimestre de gravidez é freqüente a presença do ferro sérico baixo, CTLF alto, sem haver a presença de anemia ${ }^{46}$.

Flores e col. ${ }^{13}$, estudando gestantes na província de Cartago, Costa Rica, obliveram resultados mais clevados nas médias de ferro sérico, nos três trimestres de gestação, do que as médias encontradas no presente trabalho, não havendo diferença significativa entre as médias encontradas por aqueles autores nos três trimestres gestacionais.

Szarfac e col. ${ }^{43}$, obliveram valores semelhantes aos presentes achados, nas médias da saturação da translerrina, mostrando que esta diminui de valor durante todo o processo gestacional.

Lira $\mathrm{c}$ col. ${ }^{27}$, observaram que a média da saturação da transferrina $\mathrm{cm}$ parturientes $\mathrm{em}$ Santiago,

Tabela 1. Distribuição das gestantes segundo os trimestres gestacionais e suas respectivas médias e desviospadrāo das concentraçōes de ferro sérico (FS) em $\mu \mathrm{g} / \mathrm{dl}$, capacidade total de ligação com o ferro (CTLF) em $\mu \mathrm{g} / \mathrm{dl}$ e saturação da transferrina (SAT) em \%

\begin{tabular}{cccc}
\hline Trimestre & FS $^{*}$ & CTLF* $^{*}$ & SAT* \\
\hline 1 & $105,3( \pm 32,8)$ & $325,0( \pm 51,9)$ & $30,4( \pm 8,9)$ \\
& $n=196$ & $n=196$ & $n=172$ \\
II & $90,6( \pm 33,5)$ & $368,7( \pm 52,6)$ & $24,3( \pm 9,9)$ \\
& $n=139$ & $n=133$ & $n=132$ \\
III & $62,5( \pm 26,2)$ & $440,4( \pm 66,6)$ & $17,5( \pm 10,9)$ \\
& $n=24$ & $n=28$ & $n=28$ \\
&
\end{tabular}

Na análise de variância significativa, para as très variáveis (ferro sérico, capacidade total de ligação com ferro e saturação da transferrina), houve diferença significativa entre as médias nos três trimestres gestacionais. 
Chile, foi de $18,4 \%$, semelhante a de nossa população no terceiro trimestre gestacional. Grcbc e col. ${ }^{14}$, estudando também gestantes de Santiago, Chile, obtiveram médias da saturação da transferrina nas 20 primeiras semanas de gravidez, de $29,1 \%$, e nas seguintes ( 21 a 38 scmanas), de 19,3\%.

Lamparelli e col. ${ }^{24}$, estudando gestanics de Joannesburg, África do Sul, observaram que a média da saturação da transferrina decresce com o decorrer da idade gestacional, scndo a média da saturação da transferrina no primeiro trimestre, de $23,1 \%$, no segundo, $19,1 \%$ e $14,6 \%$ no terceiro trimestre.

A prevalência de deficiência de ferro, adotando os critérios da $O M S^{15,50}$, foi de $12,4 \%$ (saluração da transferrina inferior a 15\%) e 10,2\% (dosagem de ferro sérico inferior a $50 \mu \mathrm{g} / \mathrm{dl}$ ), não havendo diferença significativa entre as freqüências obtidas por estes dois critérios.

Coulibaly e col. ${ }^{6}$, verificaram deficiência de ferro (ferro sérico $<50 \mu \mathrm{g} / \mathrm{dl}$ ) em $10 \%$ das gestantes migrantes do Norle de Cameroon, Álíica, sendo que $40 \%$ delas podiam ser consideradas deficientes se o critério fosse a saturação da transferrina $<15 \%$. Szarfac ${ }^{41}$ encontrou $15,7 \%$ de deficiência de ferro (ferro sćrico $<70 \mu \mathrm{g} / \mathrm{dl}$ ) em parturientes da Casa Matcrnal e de Assistência a Infância da Legião Brasileira cm São Paulo. Bayley e col. ${ }^{2}$ estudando gestantes de baixo nivel sócio-cconômico em primcria consulta $\mathrm{em}$ Gainsville, na Flórida, cncontrou que $4 \%$ das gestantes apresentavam (crro sérico $<50 \mu \mathrm{g} / \mathrm{dl}$ ) e $12 \%$ delas apresentavam saturação da transferrina $<15 \%$. Lira e col..$^{27}$ estudando parturientes em Santiago, Chile, observaram que $41,1 \%$ apresentavam deficiência de ferro (saturação da transferrina $<15 \%$ ), enquanto que Cardozo e col. ${ }^{4}$, cstudando gestantes em Santa Cruz, Bolívia, observaram que 59\% das gestantes cram deficientes de ferro (sideremia baixa).

No presente estudo verilicamos que das gestantes anêmicas $(n=45), 19(42,2 \%)$ apresentaram dosagem de ferro sérico < $50 \mu \mathrm{g} / \mathrm{dl}$ e 21 (46,7\%) apresentaram saturação da transferrina inferior a $15 \%$. Nas não anêmicas $(n=318), 18(5,7 \%)$ c 24 $(7,5 \%)$ apresentaram, respectivamente dosagem de ferro sérico $<50 \mu \mathrm{g} / \mathrm{dl}$ c saturação da transferrina $<15 \%$, sendo assim deficientes de ferro com ausência de ancmia (Tabcla 2).

$\mathrm{Na}$ Tabela 3 são aprescntadas as prevalências de deficiência de ferro segundo o trimestre gestacional, utilizando os dois critérios da OMS, observando-se que a prevalência de deliciência de ferro foi significativamente maior no terceiro trimestre gestacional para os dois critérios adotados. Houve diferença significativa entre os três trimestres gestacionais, quanto ao critério saturação da transferrina $<15 \%$, sendo a freqüência no pri-
Tabela 2. Distribuição das gestantes em anêmicas e não-anêmicas e suas respectivas prevalências de deficiência de ferro segundo os critérios da OMS: dosagem de ferro sérico $<50 \mu \mathrm{g} / \mathrm{dl}$ ou saturaçăo de transferrina $<15 \%$.

\begin{tabular}{lccc}
\hline & \multicolumn{2}{c}{ Deficiência de ferro } & \\
\cline { 2 - 4 } Gestantes & $\begin{array}{c}\text { Ferro sérico } \\
<50 \mu \mathrm{g} / \mathrm{dl}\end{array}$ & $\begin{array}{c}\text { Saturação } \\
\text { transferrina } \\
<15 \% \\
n(\%)\end{array}$ & $\begin{array}{c}\text { Número de } \\
\text { gestantes }\end{array}$ \\
& $n(\%)$ & $n(\%)$ \\
\hline anêmicas & $19(42,2)$ & $21(46,7)$ & $45(100,0)$ \\
não-anêmicas & $18(5,7)$ & $24(7,5)$ & $318(100,0)$ \\
\hline Total & $37(10,2)$ & $45(12,4)$ & $363(100,0)$ \\
\hline
\end{tabular}

Tabela 3. Distribuição das gestantes de acordo com o trimestre de gestação, com as respectivas prevalências de anemia e deficiência de ferro.

\begin{tabular}{|c|c|c|c|c|}
\hline \multirow[b]{2}{*}{$\begin{array}{l}\text { Trimestre } \\
\text { (número de } \\
\text { gestantes) }\end{array}$} & \multirow[b]{2}{*}{$\begin{array}{l}\text { Anemia } \\
n(\%)\end{array}$} & \multicolumn{2}{|c|}{ Deficiência de ferro } & \multirow[b]{2}{*}{$\begin{array}{c}\text { Número } \\
\text { de } \\
\text { gestantes }\end{array}$} \\
\hline & & $\begin{array}{c}\text { Ferro sérico } \\
<50 \mu g / d l \\
n(\%)\end{array}$ & $\begin{array}{c}\text { Saturaçăo } \\
\text { transferrina } \\
<15 \% \text { *** } \\
n(\%)\end{array}$ & \\
\hline $\begin{array}{l}\text { I } \\
\text { III }\end{array}$ & $\begin{array}{r}7(3,6)^{*} \\
29(20,9) \\
9(32,1)\end{array}$ & $\begin{array}{l}11(5,6) \\
16(11,5) \\
10(35,7)^{* *}\end{array}$ & $\begin{array}{r}9(4,6) \\
24(17,3) \\
12(42,8)\end{array}$ & $\begin{array}{r}196 \\
139 \\
28\end{array}$ \\
\hline
\end{tabular}

* Na análise do Qui-quadrado, a prevalência de anemia no primeiro trimestre foi significativamente menor do que as prevalências no segundo e terceiro trimestres.

* Na análise do Qui-quadrado, a prevalência de deficiência de ferro (dosagem de ferro sérico $<50 \mu \mathrm{g} / \mathrm{dl}$ ) no terceiro trimestre foi significativamente maior do que as prevalências no primeiro e segundo trimestres.

** Na análise do Qui-quadrado, a prevalência de deficiência de ferro no primeiro trimestre foi significativamente menor que a prevalência no segundo trimestre, a prevalência do segundo é menor do que a do terceiro.

meiro trimestre $(4,6 \%)$ menor do que a segundo $(17,3 \%) \mathrm{e}$ a do segundo menor do que a do terceiro trimestre $(42,8 \%)$.

Lira e col. ${ }^{26}$ encontraram $9,9 \%$ de deficiência de forro (saturação da transferrina $<15 \%$ ) em gestantes com até 20 semanas de gravidez e $34,8 \%$ de deficientes nas gestantes com mais de 20 semanas de gravidez, cm Santiago no Chile. Grebe e col. ${ }^{14}$ estudando gestantes $\mathrm{cm}$ Santiago, Chile, encontrou as seguintes deliciências de ferro (saturação da transferrina $<15 \%$ ) $\mathrm{cm} 8,2 \%$ das gestantes com até 20 semanas e $34,2 \%$ nas gestantes com idade gestacional supcrior a 20 scmanas.

Diez-Ewald e Molina ${ }^{10}$ obliveram as seguintes deficiências de ferro (ferro sérico $<50 \mu \mathrm{g} / \mathrm{dl}$ ): $22 \%$ no primeiro trimestre, $27 \%$ no segundo e $28 \%$ no terceiro trimestre, em gestantes de Maracaibo, Venczucla. 
Tabela 4. Distribuição das gestantes deficientes de ferro (FS-ferro sérico < $50 \mu \mathrm{g} / \mathrm{dl}$ e SAT-saturação da transferrina $<15 \%$ ) segundo o número de partos.

\begin{tabular}{lccc}
\hline & \multicolumn{2}{c}{ Deficiência de ferro } & \\
\cline { 2 - 3 } Número & FS $<50 \mu g / d l$ & $S A T<15 \%$ & $\begin{array}{c}\text { Número } \\
\text { de partos }\end{array}$ \\
\cline { 2 - 3 } & $n(\%)$ & $n(\%)$ & de gestantes \\
\hline nenhum & $13(8,6)$ & $16(10,6)$ & 151 \\
1 & $11(11,8)$ & $12(12,9)$ & 93 \\
2 & $4(7,7)$ & $8(15,4)$ & 52 \\
$\geq 3$ & $9(13,4)$ & $9(13,4)$ & 67 \\
\hline Total & $37(10,2)$ & $45(12,4)$ & 363 \\
\hline
\end{tabular}

Análise do Qui-quadrado não significativo, não houve diferença significativa entre as freqüências de deficiência de ferro (pelos dois critérios adotados) e o número de partos.

Tabela 5. Distribuição das gestantes segundo o intervalo do último parto em gestantes multigestas com intervalo até dois anos e multigestas com intervalo maior que dois anos e suas respectivas prevalências, deficiência de ferro - dosagem de ferro (FS) $<50 \mu \mathrm{g} / \mathrm{dl}$ ou saturação da transferrina $(\mathrm{SAT})<15 \%$.

\begin{tabular}{|c|c|c|c|}
\hline \multirow[b]{2}{*}{$\begin{array}{l}\text { Intervalo do } \\
\text { último parto }\end{array}$} & \multicolumn{2}{|c|}{ Deficiência de ferro } & \multirow[b]{2}{*}{$\begin{array}{c}\text { Número } \\
\text { de gestantes } \\
n(\%)\end{array}$} \\
\hline & $\begin{array}{c}\mathrm{FS}<50 \mu \mathrm{g} / \mathrm{dl} \\
n(\%)\end{array}$ & $\begin{array}{c}\text { SAT }<15 \% \\
n(\%)\end{array}$ & \\
\hline $\begin{array}{l}\text { até dois anos } \\
\text { maior que dois }\end{array}$ & $\begin{array}{c}17(16,7)^{*} \\
7(6,4)\end{array}$ & $\begin{array}{r}12(11,8) \\
8(7,2)\end{array}$ & $\begin{array}{l}102(100,0) \\
110(100,0)\end{array}$ \\
\hline Total & $24(11,3)$ & $20 \quad(9,4)$ & $212(100,0)$ \\
\hline
\end{tabular}

- Na análise do Qui-quadrado significativo, a prevalência de deficiência de ferro (dosagem de ferro sérico < $50 \mu \mathrm{g} / \mathrm{dl}$ ) em gestantes com intervalo do último parto até dois anos foi significativamente maior que a prevalência em gestantes com intervalo maior que dois anos.

Do total de 363 gestantes, $151(41,6 \%)$ não tiveram nenhum parto anterior àqucla gestação, 93 $(25,6 \%)$ tiveram um parto, $52(14,3 \%)$ tiveram dois e $67(18,5 \%)$ tivcram três ou mais. Não houve diferença significativa entre as freqüências de deficiência de ferro segundo o número de partos tido pela gestante (Tabcla 4).

Das gestantes que tiveram um parto ou mais (n $=212)$, $\mathrm{em} 102(48,1 \%)$ o intervalo do último parto foi de até dois anos e em $110(51,9 \%)$ cste intervalo foi maior que dois anos. A prevalência de deficiência de ferro (ferro sćrico $<50 \mu \mathrm{g} / \mathrm{dl}$ ) foi significativamente maior nas gestanles com intervalo do último parto até dois anos. Não houve diferença significativa entre as duas freqüências quando o critério adotado foi a saturação da transferrina $<15 \%$ (Tabela 5) .
Tabela 6. Prevalência de deficiência de ferro (ferro sériCo - FS < $50 \mu \mathrm{g} / \mathrm{dl}$ e saturação da transferrina - SAT < $15 \%$ ) em gestantes de primeira consulta segundo 0 salário mínimo per capita (SMPC).

\begin{tabular}{lccc}
\hline & \multicolumn{2}{c}{ Deficiência de ferro } & \\
\cline { 2 - 3 } SMPC & $\mathrm{FS}<50 \mu \mathrm{g} / \mathrm{dl}$ & $\mathrm{SAT}<15 \%$ & $\begin{array}{c}\text { Número } \\
\text { de gestantes } \\
\mathrm{n}(\%)\end{array}$ \\
\hline$\leq 0,5$ & $14(13,9)$ & $20(19,6)^{* *}$ & $102(100,0)$ \\
$0,541,0$ & $12(8,9)$ & $13(9,7)$ & $134(100,0)$ \\
$>1,0$ & $11(9,0)$ & $12(9,8)$ & $122(100,0)$ \\
\hline Total & $37(10,3)$ & $45(12,6)$ & $358(100,0)$ \\
\hline
\end{tabular}

- Cinco gestantes não declararam renda.

* Na análise do Qui-quadrado, a prevalência de deficiência de ferro (saturação da transferrina < 15\%) em gestantes pertencentes à familia com renda per capita até 0,5 SMPC foi significativamente maior em relação as demais rendas.

A prevalência de deficiência de ferro foi significativamente maior naquelas gestantes pertencentes a famílias com renda per capita de até 0,5 SMPC (Tabela 6).

A infecção por ancilostomídeos pode ocasionar a perda de sangue de modo crônico levando a deficiência de ferro. A prevalência de infecção por ancilostomídeos foi de $14,0 \%(\mathrm{n}=300)$, semelhante à encontrada por $\mathrm{Vaz}$ Pinto e col. ${ }^{45}$ Nas gestantes deficientes de ferro (saturação da transferrina < $15 \%)$ apcnas $5(11,1 \%)$ estavam infectadas por este helminto, $11(24,4 \%)$ tiveram o exame protoparasitológico negativo e $10(22,2 \%)$ não trouxeram amostra de fezes para análise. Estes resultados sugerem que a infecção por ancilostomíneos não pode ser considerada um fator que tenha influenciado na eliologia da deficiência de ferro.

O Grupo de Pcritos da Organização Mundial da Saúde ${ }^{50}$ cita que na concentração da hemoglobina corpuscular média (CHCM) inferior a $31 \%$ sugere a presença de deficiência de ferro. No presente trabalho, observou-se que o CHCM foi inferior a 31 $\mathrm{em} \mathrm{16,2 \%} \mathrm{das} \mathrm{gestantes} \mathrm{com} \mathrm{ferro} \mathrm{sérico}<50 \mu \mathrm{g}$ / dl c $13,3 \%$ daquelas com saturação da transferrina $<15 \%$, sugerindo que o CHCM não é um bom indicador para deficiência de ferro.

Nas gestantes com hemoglobina $<11,6 \mathrm{~g} / \mathrm{dl}$ ( $\mathrm{n}$ $=45$ ) foram encontradas as seguintes deficiências: $46,7 \%$ de ferro, $44,4 \%$ de ácido fólico, $20,0 \%$ deliciente concomitantemente de ferro e ácido fólico e nenhuma gestante deficiente $\mathrm{cm}$ viLamina $\mathrm{B}_{12}$ (Tabcla 7).

A menor concentração sérica de vitamina $B_{12}$ encontrada foi de $138 \mathrm{pg} / \mathrm{ml}$ em uma das gestantes anêmicas. Esta gestante teve dois partos 
Tabela 7. Etiologia das anemias, de acordo com as deficiências de: ferro, ácido fólico, vitamina $\mathrm{B}_{12}$ e associação de deficiência em ferro e ácido fólico.

\begin{tabular}{|c|c|c|c|}
\hline \multirow[t]{2}{*}{ Deficiências } & \multirow[t]{2}{*}{ Critérios } & \multicolumn{2}{|c|}{ Freqüência } \\
\hline & & $n$ & $\%$ \\
\hline \multirow[t]{2}{*}{ Ferro } & $\begin{array}{l}\text { ferro sérico }<50 \mu \mathrm{g} / \mathrm{dl} \\
\text { saturação da transferrina }<15 \% \\
\text { ferro sérico }<50 \mu \mathrm{g} / \mathrm{dl} \text { e }\end{array}$ & $\begin{array}{l}19 \\
21\end{array}$ & $\begin{array}{l}42,2 \\
46,7\end{array}$ \\
\hline & saturaçāo de transferrina $<15 \%$ & 18 & 40,0 \\
\hline Ácido fólico & ácido fólico sérico $<3 \mathrm{ng} / \mathrm{ml}$ & 20 & 44,4 \\
\hline Vitamina $\mathrm{B}_{12}$ & vitamina $\mathrm{B}_{12}<80 \mathrm{pg} / \mathrm{dl}$ & 0 & 0 \\
\hline $\begin{array}{l}\text { Ferro e } \\
\text { ácido fólico }\end{array}$ & $\begin{array}{l}\text { saturação da transferrina }<15 \% \\
\text { e ácido fólico }<3 \mathrm{ng} / \mathrm{ml}\end{array}$ & 9 & 20,0 \\
\hline
\end{tabular}

anteriores sendo o intervalo do último de um ano e sete meses, cstava no terceiro trimestre de gestação, apresentou concentração de ferro sérico igual a $61 \mu \mathrm{g} / \mathrm{dl}$, saturação da trasferrina de $10,9 \%$ e ácido fólico de $4,1 \mathrm{ng} / \mathrm{ml}$. As demais gestantes apresentaram níveis de vilamina $B_{12}$ superiores a $140 \mathrm{pg} / \mathrm{ml}$. Se forem consideradas deficientes $\mathrm{em}$ vitamina $\mathrm{B}_{12}$ aquelas gestantes com dosagem séricas até $140 \mathrm{pg} / \mathrm{ml}{ }^{45,50}$, tor-scia 2,2\% de deficiência desta vilamina.

Vaz Pinto e col. ${ }^{45}$ oblivcram as seguintes deliciências em gestantes anêmicas da cidade salélite de Sobradinho, Brasília, DF: $73,7 \%$ de ferro, $26,3 \%$ de ácido fólico (concentração sérica $<4,0$ $\mathrm{ng} / \mathrm{ml}$ ), $10,5 \%$ de vitamina $\mathrm{B}_{12}$ (concentração sérica desta vitamina $<140 \mathrm{pg} / \mathrm{ml}$ ). Hercberg $e$ Galan ${ }^{19}$ estudando gestantes $\mathrm{cm}$ duas maternidades públicas em Benin, África, encontraram que a anemia estava associada a deficiência de ferro em $83 \%$ e a deficiência de ácido lólico $\mathrm{cm} 48 \%$. Loria e col. ${ }^{28}$ obscrvaram que as gestantes ancmicas, na cidade do México, aprescntaram as scguintes deficiências: $47 \%$ de ferro, $6 \%$ de lerro e ácido fólico, $7 \%$ de ácido fólico e $1 \%$ de vilamina $\mathrm{B}_{12}$. Dicz-Ewald e Molina ${ }^{10}$, estudando 132 gestantes $\mathrm{cm}$ Maracaibo, Venczuela, não encontrou nenhuma gestante com concentração sérica de vitamina $B_{12}<80 \mathrm{pg} / \mathrm{ml}$, sendo que nas gestantes anêmicas relataram as seguintes deficiências: $17 \%$ de ferro, $11 \%$ de ácido fólico c $30 \%$ de ferro e ácido fólico concomilantementc.

A alta frequêencia de deficiência de ácido fólico no grupo das gestantes anêmicas (44\%) é superior à encontrada por outros autores no Brasil ${ }^{45}$ e na América Latina ${ }^{10,28}$, regiões onde o quadro sócioeconômico não deve ser muito diferente.

Incidência tão elevada de deficiência de ácido fólico nas grávidas $\mathrm{tcm}$ sido relatado $\mathrm{cm}$ grupos populacionais mais pobres como India ${ }^{52}$ ou na África ${ }^{19}$. Também Molina ${ }^{34}$ encontrou $\mathrm{cm}$ seu trabalho, na Verezucla, que o grupo de gestantes que recebcu tratamento profilático com ferro teve maior incidência de ácido fólico baixo no soro. $\mathrm{O}$ autor sugere, para explicar este fato, que a administração de ferro incrementaria a critropoese causando aumento no consumo de ácido fólico. As pacientes incluídas no presente cstudo não faziam uso de ferro prolilático.

Os presentes resultados, principalmente da alta freqüência de deficiência de ácido fólico, indicam a necessidade de outros estudos com população de gcstantcs, contribuindo para rcavaliar medidas de prevenção de ancmia no Programa de Atendimento à Gestante da rede pública.

\section{Agradecimentos}

A Dirctoria do SUDS-RO2, aos diretores e aos funcionários dos Centros de Saúdc: Escola Butantan, II do Butantan, São Luiz, Rio Peçueno, Vila Borges, Caxingui, Monte Kemel c Real Parque.

A Seção de Coleção de Culluras do Instituto Adolfo Luk (IAL), na pessoa da Dra. Marina Ortolan, pelo fornecimento das cepas dos bacilos utilizados neste trabalho.

Às colegas Iara Ribciro, Judic de Oliveira Bras e Ana Lúcia Zavitoski, da Seção de Hcmatologia do IAL, pelo auxílio técnico neste trabalho.

GUERRA, E. M. et al. [The prevalence of iron deficiency in pregnant women at their first consultation in licalth centers in a metropolitan area, Brazil. Etiology of anemia in anemic pregnant women]. Rev. Saúde pübl. S. Paulo, 26: 88-95, 1992. Three hundred and sixly-three pregnant women enrolled in the Pregnancy Medical Care Program of S. Paulo Heallh Department in the district of Butantan, S. Paulo cily, Brazil, were studied at their first routine consultation between April and October, 1988. Their average age was 25 and $65,9 \%$ of them belonged to families with a monthly income below US\$ 50.00 per capila. Only $3.1 \%$ presented an income above US\$ 150.00 per capita. Taking the minimum transferrin saturation threshold of $15 \%$ as determining iron deficiency, a $4.6 \%$ prevalence of iron deficiency was observed in the first trimester, $17.3 \%$ in the second trimester and $42.8 \%$ in the third trimester, resulting in an overall prevalence of $12.4 \%$. There was no significant difference between prevalences of iron deficiency according to the number of pregnancies. The prevalence of iron deficiency was higher in women presenting incomes below USS 50.00 per capita.

Keywords: Iron deficiency, epidemiology. Pregnancy complications. Anemia hypochromic, ctiology. 


\section{Referênclas Bibliográticas}

1. ALMEIDA, P. A. M. de; CIARI JR., C.; SANTOS, J. L. I.; SIQUERRA, A. A. F. de. Curva de hemoglobina $\mathrm{cm}$ um grupo de gestantes normais. Rev. Saúde públ., S. P’aulo, 7: 273-82, 1973 .

2. BAYLE, L. B.; MAIIAN, C. S.; DIMPERIO, D. Iolacin and iron status in low-income pregnant adolescents and mature women. Amer. J. clin. Nutr., 33: 1997-2001, 1980.

3. BEALE, R. N.; BOSTROM, J. O.; TAYLOR, R. F. Improved rapid methods for the deternination of iron content and binding capacity of scrum. J. clin. Pathol., 15: $156-60,1962$.

4. CARDOZO, L.; ZUNA, II.; URJEL, R.; SAAVEDRA, F. Estudio de las causas de ancmia en cmbarazadas de la maternidad Percy Boland de la ciudad de Sanla Cruz - 1985 Bol.cient. CENETROP, 11:58-67, 1985. [Separata].

5. CIIANARIN, I. Iron deficiency in pregnancy and possible interrelatio with folate. In: Intemational Congress of Hematology, 149, São Paulo, 1972. p. 7.

6. COULIBALY, M.; COSIAGLIOLA, D.; ZITIOUN, J.; MARY, J. Y. Modification of hemalo-biological parameters in pregnant women in a migrating population in Northern Cameroon; prevalence of anemia, iron and folates deficiences. Int. J. Vitam. Nutr. Res., 57: 173-8, 1987.

7. DACIE, J. V. \& LEWIS, S. M. Praclical haematology. 6th ed. Edinburgh, Churchill Livingstonc, 1984.

8. DAWSON, E. B. \& MCGANII'Y, W. J. Protection of matemal iron stores pregnancy. J. Reprod. Med., 32 (6 suppl.): 478-87, 1987.

9. DEWEES, C.B. Ilematologic disorders in pregnancy. Nurs. clin. North Amer., 17: 57-67, 1982.

10. DIEZ-LWALD, M. \& MOLINA, R. A. Iron and folic acid deficiency during pregnancy in Western Venezuela. Amer. J. trop. Med.Ilyg., 21: 587-91, 1972.

11. FAVIiLAS do Município de São Paulo. Diário oficial do Município, S. Paulo, 2 dez. 1986. p. 9.

12. FINCII, C. A. Red cell manual. Seatte, University of Washington, 1969. p. 9.

13. FLORES, M. E.; RODRIGUES, J.; SANTTSTEIBAN, I.; ARAUZ, A. G.; CLSPEDES, C. Un problema nutricional active la deficiencia de hicro y anemia en la mujer cmbarazada. Rev. costarric. Cienc. med., 5(1): $52-60,1984$.

14. GREBE, G.; LIRA, P.; ARTEAGA, A.; VALENZULiLA, M.; CUBILLOS, A. M.; I:ORADORI, A. Corrclacionentre la deficiencia de hierro y la ingesta dietelica de hierro en cl cmbarazo. Rev. med. Chile, 107: 989-92, 1979.

15. GRUPO MIXTO FAO/OMS DE EXPIERI'OS EN NECESIDADES DE ÁCIDO ASCORIBICO, VI'IAMINA D, VITAMINA B ${ }_{12}$. IOLLA'OO y IIIERRO, Ginebra, 1969. Informe. Gincbra, 1971. (O.MS - Serie de Informes Técnicos, 452)

16. GUERRA, E. M. Prevalência de anemia $\mathrm{cm}$ gestantes de primcira consulta em Centros de Saúde do Estado no Subdistrito de Paz do Butantan, município de São Pau1o. São Paulo, 1989. [Dissentação de Mestrado - laculdade de Ciências Farmacêticas da USI'].

17. GUIERRA, E. M.; I3ARRLI'TOO, O. C. de; VA\%, ^. J.; SILVEIRA, M. B. Prevalência de ancmia em gestantes de primcira consulta em Centros de Saúde de área metropolitana, Brasil. Rev. Saúde públ., S. Paulo, 24: 380-6, 1990.

18. HERBERT, V. Recommended dietary intakes (RDI) of iron in humans. Amer. J. clin. Nurr., 45: 679-86, 1987.

19. HERCERBERG, S. \& GALAN, P. Nutritional anacmia in pregnant Benincse women: consequences on the haematological profite of the newbom. Brit. J. Nutr., 57: 185-93, 1987.

20. HOFIMAN, W. A.; PONS, J. A.; JANER, J. L. The sedimentation concentration method in Schistosomiasis mansoni. Puerto Rico J. publ., Illth trop. Med., 9: 28398, 1934.

21. HUR'IADO, W. A.; MERINO, C.; DELGADO, E. Influence of anoxemia on the hemopoictic activity. Arch. intern. Med., 75: 284-323, 1945.

22. INITERNATIONAL COMMI'IEE FOR STANDARDIZATION LN IIALMATOLOGY. Proposed recommendations for measurements of serum iron in human blood. Amer. J.clin. Pathol., 56: 543-5, 1971.

23. KOMIYA, Y. \& KOBAYASIII, A. Iivaluation of Kato's tick-smear technique with a cellophane cover for helminth eggs in feces. Jap. J. med. Sci. Biol., 19: 59-64, 1966.

24. LAMPARELLI, R. D. V.; BOTHIWELL, T. II.; MACPIIAIL, A. P.; WANDER, W. J.; BAYNES, R.D.; MACFARLANE, B. J. Nutritional anemia in pregnant coloured women in Johannesburg. S. Afr. Med. J. 73: 477-81, 1988.

25. LANGE, R. D. \& DYNESIUS, R. Blood volume changes during nornal pregnancy. Clin. Haematol., 2: 433.51, 1973.

26. LIRA, P.; FOORADORI, A.; GREBE, G; LLGUE, M. E.; MUNIIO\%, B.; AR'TEAGA, A.; VELA, P. Características hematológicas de una poblacion de embarazadas en Chile. Rev. med. Chile, 106: 343-9, 1978.

27. L.IRA, P.; I:ORADORI, A.; GREBE, G.; VELA, P. Deficiencia de hicro y folato en mujeres embarazadas de termino. Rev. med. Chile, 112: 127-31, 1984.

28. I.ORI $\Lambda$, ᄉ.; ARIROYO, P.; PILDRAS, J.; MLDAL, L. S. Ancmia en el embarazo. Il. Datos hematologicos y obstretricos en embarazadas de dos instituciones mexicanas. Rev. Invest. clin., 31: 217-30, 1979.

29. MAR'IINS, I. S.; ALVARLENGA, A. 'I'; SIQUEIRA, A. A. F.: SZARFARC, S. C.: LIMA, F. D. As determinaçöes biológica c social da doença: um estudo de anemia ferropriva. Rev. Saúde públ., S. Paulo, 21: 73-89,1987.

30. MASPI:S, V.; LUTIIOLD, W. M.; NAGAI, M. A.; MARLET, J. M. Dosagem de ferro sérico e da capacidade de transporte de ferro pela transferrina. Rev. Hosp. Clin. Fac. Med. Univ. S.Paulo, 37: 5-9, 1982.

31. MATILIISWS, D. M. Observations on the estimation of serum vitamin $B_{12}$ usilly Laclobaccillus leichmannii. Clin. Sci., 22: 101-11, 1962.

32. McIFLE, J. G. Iron metabolism and deficiency during pregnancy. Clin. Obstet. Gynec., 22: 799-817, 1979.

33. MCGANII'Y, W. J. Protection of maternal iron stores in pregnancy. J. Reprod. Med., 32 (6 Suppl.): 475-7, 1987.

34. MOLINA, R.A.; DII:Z-EWAI.D, M.; FERNANDEZ, G.; VIELAKQUL\%, N. Nutritional anacmia during pregnancy: a comparative study of two socio-economic classes. J. Obstet. Gynec, 81: 454-8, 1974.

35. MORAIS, E. N. Repercussōes da anemia no ciclo gravídico puerperal e sobre o recém-nascido. Bol. Soc. bras. Ilemalol. llemoler., 10 (149): 140-3, 1988.

36. PICCK, T.M. \& ARIAS, F. Hematologic changes associated with pregnancy. Clin. Obstet. Gynec., 22: 785-98, 1979.

37. RONCADA, M. J. \& SZARIARC, S. C. IIipovitaminose A e ancmia ferropriva em gestantes de duas comunidades do vale do Ribcira (Estado de São Paulo, Brasil). Rev. Saúde públ., S. Paulo, 9: 99-106, 1975.

38. ROSENIIELD, L. G. M. \& SIGULEM, D. M. Recomendações profiláticas e terapêuticas na anemia. Bol. Soc. bras. Llematol./lemoter., 10(149): 177-80, 1988. 
39. SECRETARIA DE ESTADO DA SAÚDE. Grupo de Trabalho. Implantação do ERSA 2; rclatório. São l’aulo, 1986. [Mimeografado].

40. SECRETARIA DE ESTTADO DA SAÚDE. Grupo de Saúde da Mulher. Sub-programa de Saíde da Mulher: prénatal normal. São Paulo, 1988. v. 2.

41. SZARFARC, S. C. Ancmia ferropriva em parturientes e recém-nascidos. Rev. Saúde públ., S. Paulo, 8: 369. $74,1974$.

42. SZARFARC, S. C.; SIQUEIRA, A. A. F; MARTINS, I. S.; TANAKA, A. C. D'A. Estudo comparativo de indicadores bioquímicos da concentração de ferro, $\mathrm{cm}$ duas populações de gestantes, com e sem atendimento de prénatal. Rev. Saúde públ., S. Paulo, 16: 1-16, 1982.

43. SZARFARC, S. C.; SIQUEIRA, A. A. F.; MAR'TINS, I. G. Avaliação da concentração de ferro orgânico cm uma população de grávidas. Rev. Saúde públ., S. Paulo, 17: 200-7, 1983.

44. VAZ PINTO, A.; SANTOS, F.; MIDLEJ, M. C.; ALMEIDA, A. M.; GAMA, M. P. Vitamin $B_{12}$ and acid folic in maternal and newborn scra. Rev. Invest. clin., 25: 341 4,1973.

45. VAZ PINTO, A.; PINTO, G. P.; FORMIGA FILIIO, J. F. N.; LARA, S.; SANTOS, F. A ancmia da gravidez em Sobradinho, cidade satélite de Sobradinho, Brasil. Rev. bras. Pesq. méd., 8: 381-5, 1975.
46. WALLERSTELN, R. O. Iron metabolism and iron deficiency during pregnancy. Clin. Ilaematol., 2: 453-60, 1973.

47. WATERS, A. H. \& MOLLIN, D. L. Studies on the folic acid activity of human serum. J. clin. Palhol., 14: 335 44, 1961.

48. WILLIS, H. II. A simple levitation method for the detection of hookworm ova. Med. J. Aust., 29: 375-6,1921.

49. WORLD IILALTHI ORGANIZATION. Scientific Group on Nutritional Anacmias, Geneva, 1967. Report. Geneva, 1968. (Technical Report Series, 405).

50. WORLD IIEALTHI ORGANIZATION. Group of Experts on Nutritional Anemias, Geneva, 1971. Report. Geneva, 1972. (Technical Repon Series, 503).

51. YAZLLE, M. E. H. D. Adaptações hematológicas do organismo matemo. Bol. Soc. bras. Hematol. Hemoter., 10 (149): 137-9, 1988.

52. YUSURJI, D.; MATHAN, V.I.; BAKER, S.J. Iron folate and vitamin $B_{12}$ nutrition in pregnancy: a study of 1000 women from southem India. Bull. Wld Hlth Org., 48: $15-22,1973$.

Recebido para publicaşão em 17/6/1991 Reapresentado em 2/1/1992 Aprovado para publicação em 10/2/1992 